\title{
Morfometria de Papilioninae (Lepidoptera, Papilionidae) ocorrentes em quatro localidades do Rio Grande do Sul, Brasil. II. Relação entre partes do corpo, aerodinâmica de vôo e tipos de asas
}

\author{
Rocco Alfredo Di Mare ${ }^{1} \&$ Elio Corseuil ${ }^{2}$ \\ ${ }^{1}$ Departamento de Biologia, Centro de Ciências Naturais e Exatas, Universidade Federal de Santa Maria. Campus \\ Universitário, Faixa de Camobi, km 9, 97115-900 Santa Maria, Rio Grande do Sul, Brasil. E-mail: ram13@ terra.com.br \\ ${ }^{2}$ Curso de Pós-Graduação em Biociências, Pontifícia Universidade Católica do Rio Grande do Sul. Caixa Postal 1429, \\ 90619-900 Porto Alegre, Brasil. E-mail: corseuil@pucrs.br
}

\begin{abstract}
Morphometrics of Papilioninae (Lepidoptera, Papilionidae) occurring in four communities from Rio Grande do Sul, Brazil. II. Relationships among body parts, flight aerodynamics and wing types. This study investigated morphometric parameters associated to flight in Papilioninae. Comparisons were accomplished with body parts, wing form and dynamics of flight of 10 species collected at four different places. Was analyzed the total mass of the body, thoracic mass, length and width and area of fore and hind wings, wing loading and aspect ratio and flight speed prevision. The analyses showed significative differences among species, sexes and communities investigated. The correlations between the length and width of fore wings were positive and significant for both sexes. The values obtained for wing loading, aspect ratio, and estimated flight speed, among sexes and species, were similar to those measured in the field or insectary.

KEY WORDS. Aspect ratio, body size, butterflies, insect, wing loading.
\end{abstract}

RESUMO. Neste estudo foram investigados parâmetros morfométricos associados ao vôo de Papilioninae. Foram realizadas comparações com partes de corpo, forma de asa e dinâmica de vôo de 10 espécies coletadas em quatro localidades diferentes. Analisou-se a massa total do corpo, massa torácica, comprimento, largura e área das asas anteriores e posteriores, carga unitária e índice de estreiteza e previsões da velocidade de vôo. As análises mostraram diferenças significativas entre espécies, sexos e comunidades investigadas. As correlações entre o comprimento e largura das asas anteriores foram positivas e significantes para ambos os sexos. Os valores obtidos a carga unitária, índice de estreiteza e velocidade de vôo, entre sexos e espécie, foram semelhantes àqueles medidos em campo ou insetário.

PALAVRAS CHAVES. Borboletas, carga unitária, índice de estreiteza, insetos, tamanho do corpo.

Estudos sobre a aerodinâmica e as forças necessárias ao vôo de organismos alados são fundamentais para explicar algumas adaptações e/ou comportamentos exibidos na natureza. Muitos destes estudos com insetos foram conduzidos através de vôos controlados em túneis de vento (Weis-Fogh 1956, Vogel 1966, Dudley \& Ellington 1990, Ellington et al. 1990), insetários (Eluington 1984, EnNos 1989, Dudley 1990) ou através de observações diretas na natureza (DeVRies \& Dudley 1990, Dudley \& DeVries 1990, Dudley \& SRygley 1994).

Alguns resultados obtidos sobre a velocidade dos insetos, embora limitados, podem ser verificados em LewIs \& TAYLOR (1967) e Johnson (1969). Outros estudos mais recentes foram fundamentados a partir de observações em campo (WALOFF 1972, Balciunas \& KNopf 1977, BaKer et al. 1981, BetTs \& Wootton 1988).
Apesar da influência da mecânica de vôo e do desempenho dos organismos não serem ainda completamente conhecidos, pode-se verificar uma ligação íntima entre a morfologia e a cinemática das asas e entre as forças aerodinâmicas e os mecanismos musculares de vôo (Chai \& Millard 1997, Chai \& Dudley 1995, Dudley 2000). A forma e o tamanho da asa têm em geral um compromisso com as várias funções em que elas estão envolvidas. Nas borboletas, por exemplo, as asas podem ser usadas durante a corte, na manutenção de territórios, podem estar envolvidas na defesa (camuflagem ou aposematismo), na termorregulação (KingSOLVER 1985) e na amplitude dos padrões de vôo (BAKER 1972, DAVIES 1978). Borboletas palatáveis, que usam o vôo para escaparem de predadores aéreos, investem uma grande porção da sua massa corporal na musculatura 
de vôo, ao contrário do que fazem as borboletas impalatáveis (MARDEN \& C HAI 1991). Este aumento na musculatura também foi constatado em beija-flores e machos de libélulas, quando estão engajados em combates aéreos por territórios para acasalamento (HARTMAN 1961, MARDEN 1989, MoRGAN et al. 1985). Deste modo, a resposta à sel eção natural poderia ocorrer através da alteração do "design" do corpo, de modo que a musculatura utilizada aumente proporcional mente com a massa corporal, mantendo a performance aproximadamente igual ( $\mathrm{CHAl}$ \& Dudley 1995, Chal et al. 1997).

Normalmente, são utilizados dois parâmetros para caracterizar o vôo de um objeto ou de um organismo: a carga unitária da asa e o índice de estreiteza (DudLey 2000). No entanto, alguns autores colocam restrições para o uso com insetos (Norberg 1990, Tennekes 1996, Chal \& Millard 1997, Betts \& Wootton 1988), alegando que estes parâmetros quantificam grosseiramente o tamanho e a forma da asa. DudLey (1990) mostrou haver uma correlação positiva entre a velocidade de vôo e o comprimento das asas. SRYGLEY \& CHAI (1990) também obtiveram uma correlação positiva entre a velocidade de vôo e a massa do tórax, mas negativa com a massa do abdômen (que contém os órgãos reprodutivos e armazenamento de gorduras).

Alguns comportamentos associados ao vôo das borboletas, como o tipo de vôo ou ocorrência em determinadas áreas, fazem com que estas também possam servir como boas indicadoras de alterações ambientais provocadas pela urbanização (BLAIR \& LAUNER 1997). Alguns pesquisadores (GILBERT 1980, 1984, Pyle 1980, Murphy et al. 1990, Brown Jr. 1992, Kremen 1992) propõem que elas podem avaliar indiretamente esta variação ambiental, por causa da sua sensibilidade às condições locais como clima, níveis de luminosidade (W ATT et al. 1968, EHRLICH et al. 1972, WEISs et al. 1987, 1988) e proporção da cobertura vegetal (SchwARTZ \& DI MARE 2001). Outros estudos estabelecem uma relação entre a habilidade de dispersão dos insetos com a morfologia dos adultos (Palmer \& Dingle 1989, FAIRBAIRN \& RoFf 1990).

Este trabal ho tem como objetivo estudar o relacionamento existente entre: a) partes do corpo (massa total e torácica), b) componentes da aerodinâmica de vôo (carga unitária, índice de estreiteza e previsões da velocidade) e c) forma das asas (comprimento, largura e área das asas anteriores e posteriores), entre os sexos e entre espécies de borboletas da subfamília Papilioninae coletadas em quatro localidades do município da Santa Maria, Rio Grande do Sul.

\section{Á reas de estudo}

\section{MATERIAL E MÉTODOS}

O estudo foi realizado em quatro localidades do Rio Gran-

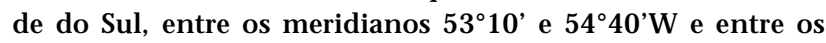
paral elos $29^{\circ} 00^{\prime}$ e $29^{\circ} 20^{\prime} \mathrm{S}$. As local idades de Água Negra (AN), Morro do Link (ML), Rincão do Canto (RC), Rincão do Soturno (RS), e Vale do Diabo (VD), estão em áreas remanescentes da floresta decidual e semidecidual da Serra Geral, com altitu- de inferior a $500 \mathrm{~m}$. Podem ser observados cinco extratos vegetais: emergente, arbóreo contínuo, arvoretas, arbustivo e herbáceo (KLEIN 1983). Schöenstatt (SC) e Camobi (CB), são áreas eminentemente urbanas, com flora caracterizada por plantas ornamentais cultivadas como: Apocynaceae, Asteraceae, Malvaceae, Myrtaceae e Verbenaceae. Schöenstatt apresenta grande variedade de flores e Camobi poucas, porém abundantes em número.

As áreas investigadas foram agrupadas segundo SCHWARTZ $\&$ DI M ARE (2001), de acordo com a cobertura vegetal presente. Deste modo, foram identificados dois grandes grupos. O primeiro formado por áreas local izadas em zonas urbanas, denominado SCCB (SC + CB). O segundo constituído por dois subgrupos: um inclui áreas de floresta, com cobertura vegetal entre $50 \%(R S M L=R S+M L)$ e $70 \%(V D R C=V D+R C)$ e o outro formado por AN, cuja cobertura é de cerca de $30 \%$. Água Negra se distinguiu das demais áreas por suas características fisionômicas particulares: hábitat fragmentado com vegetação lenhosa baixa e de média altura, observada nos capões, com abundância de Myrtaceae e Rubiaceae. Neste trabalho, os grupamentos obtidos em ScHWARTZ \& DI MARE (2001) passam a ser referidos como comunidades, por conveniência e por afinidade com a cobertura vegetal.

Em AN, RC, RS e VD, as coletas foram realizadas em triIhas e estradas existentes, previamente escolhidas. Em CB foram visitados al guns jardins em ruas percorridas no bairro, escolhidas pelo número de casas com jardins apresentando flores. Em SC foi investigada uma pequena mata secundária, com cerca de $5000 \mathrm{~m}^{2}$.

\section{Material estudado}

No presente trabalho foram estudadas dez espécies de Papilionidae, uma das quais com três subespécies, pertencentes a três tribos de Papilioninae: Leptocircini Kirby, 1896 [ = Graphiini Talbot, 1939] (Mimoides lysithous eupatorion (Lucas, [1859]); M imoides Iysithous Iysithous (Hübner, [1821]); Mimoides Iysithous rurik (Eschscholtz, 1821)), Papilionini (Heraclides astyalus astyalus (Godart, 1819); Heraclides hectorides (Esper, 1794); Heraclides thoas brasiliensis (Rothschild \& Jordan, 1906); Pterourus scamander scamander (Boisduval, 1836)) e Troidini (Battus polydamas polydamas (Linnaeus, 1758); Battus polystictus polystictus (Butler, 1874); Parides agavus (Drury, 1782); Parides anchises nephalion (Godart, 1819); Parides bunichus perrhebus (Boisduval, 1836).

\section{A mostragem e identificação}

As coletas ocorreram de setembro de 1994 a março de 1995 e de novembro de 1995 a fevereiro de 1996. Foram realizadas em interval os médios de trinta dias para cada localidade estudada, utilizando duas redes entomológicas convencionais, durante intervalos médios de quatro horas. As coletas eram iniciadas entre novee dez horas da manhã. Os adultos coletados foram acondicionados em envelopes de papel vegetal, individualizados, devidamente etiquetados e transportados para o 
Laboratório de Biologia Evolutiva do Departamento de Biologia da Universidade Federal de Santa Maria (UFSM). A identificação das espécies foi realizada com base em TYLER et al. (1994).

\section{Obtenção dos dados}

O trabalho foi fundamentado em 527 machos e 213 fêmeas, embora alguns indivíduos não tenham sido utilizados em todas as análises. As diferentes partes dos indivíduos foram separadas com uma tesoura cirúrgica de ponta fina e depois pesadas em uma balança analítica com precisão de até $0,1 \mathrm{mg}$. As pesagens foram realizadas após o trabalho de campo, com interval os de aproximadamente quatro horas entre a captura e a pesagem de cada indivíduo. Foram realizadas duas modalidades de pesagem: com os adultos íntegros ( $m$ ), os adultos sem as asas e somente o abdômen. Os valores para o tórax (mt) (incluindo cabeça e pernas) foram obtidos por diferença entre o indivíduo sem asas menos o abdômen.

As medidas do comprimento, largura e área das asas foram obtidas através de uma mesa digitalizadora, após serem montadas entre duas folhas de material plástico auto-adesivo transparente, utilizando o programa SIGDER (gentilmente cedido pelo Departamento de Engenharia Rural da UFSM). Com alfinetes entomológicos foram marcados pontos fixos nas asas, utilizados para medir o comprimento e a largura das mesmas (Fig. 1). O comprimento da asa anterior ( $\mathrm{Ca}$ ) foi medido entre o limite final da veia radial $R_{3}$ e a origem da veia anal $2 A$ e a largura (La) entre o limite final da veia anal $2 \mathrm{~A}$ e a margem costal, passando pela origem da veia radial $R_{1}$. O comprimento na asa posterior $(\mathrm{Cp})$ foi medido entre o início da fusão das veias subcostal $S_{1}$ e veia radial $R_{1}$ e o limite terminal da veia mediana $M_{2}$. A largura (Lp) entre o limite final da fusão das veias subcostal $\mathrm{Sc}_{1}$ e $\mathrm{R}_{1}$ e o limite final da veia anal $2 \mathrm{a}$. As áreas das asas ( $\mathrm{S}=$ anterior +posterior) foram medidas usando o contorno total das asas.

Para analisar a aerodinâmica das asas foram estimadas as cargas unitárias $(\mathrm{Cu}=\mathrm{m} / \mathrm{S} ; \mathrm{m}=$ massa do corpo; $\mathrm{S}=$ área das asas) e o índice de estreiteza ( $E=4 R^{2} / S ; R=$ envergadura das asas $=2 \mathrm{C}_{\mathrm{a}} ; \mathrm{S}=$ superfície das asas).

As medidas e cálculos dos parâmetros investigados foram real izados conforme o trabal ho de BetTs \& Wootton (1988). Foram realizadas também previsões da velocidade utilizando como estimador o índice de estreiteza, cuja fórmula $\left(V_{E}=46,9\right.$ $E^{-2,27}$ ) foi auferida do trabal ho de DudLEY \& SRYGLEY (1994).

\section{A nálise dos dados}

As análises estatísticas foram realizadas com base nas medidas obtidas conforme descrição no item anterior utilizando os dados log-transformados, quando não satisfaziam os critérios de normalidade e homocedasticidade.

As médias foram confrontadas através dos testes de comparações múltiplas “Least Square Significant” (LSD) e Bonferroni. Estes métodos de comparação levam em conta, especificamente, a utilização de mais de duas amostras. O primeiro baseia-se no número de exemplares dos grupos envolvidos na compara-
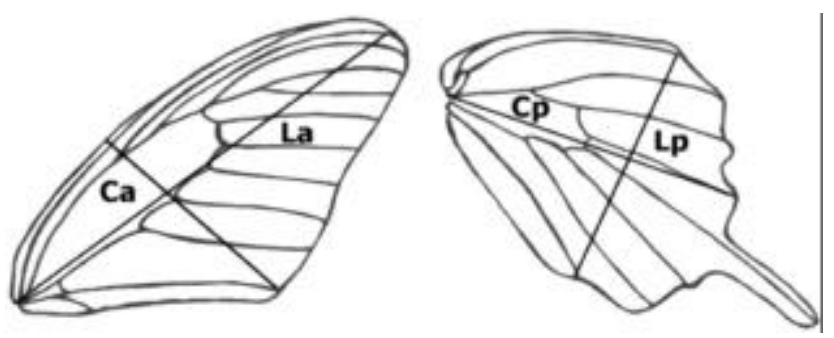

Figura 1. Pontos utilizados para estimar o comprimento (C) e a largura (L) da asa anterior (a) e posterior (p) das 10 espécies de Papilioninae.

ção. Ele oferece proteção mínima em relação a um aumento na taxa de erro alfa, devido às comparações múltiplas "a posteriori" (ZAR 1999). O segundo ajusta o nível alfa de cada teste individual para assegurar que o risco global para vários testes permaneça 0,05, diminuindo a probabilidade de ocorrer um erro do tipo I.

A análise de grupo foi realizada com o índice de estreiteza, a carga unitária e a área alar. Como as variáveis apresentam dimensões diferentes, foram empregados os percentuais relativos de cada variável para cada espécie analisada. Os grupamentos foram obtidos através do método de W ARD (W ARD 1963). Este método usa uma análise da variância para avaliar a distância entre os grupos. Ele tenta minimizar a soma dos quadrados de quaisquer dois grupos que podem ser formados a cada passo. Como medida de comparação foi utilizada a distância Manhattam.

\section{RESULTA DOS E DISCUSSÃ O}

\section{Medidas morfológicas}

A tabela I, com base em 490 machos e 187 fêmeas, mostra os val ores obtidos para a massa total $(\mathrm{m})$ (366 machos; 136 fêmeas), massa torácica ( $\mathrm{mt}$ ) (490 machos; 187 fêmeas), área das asas (S) (319 machos; 128 fêmeas) e respectivos erros padrões (ep), por espécie e comunidade estudada. Pterourus scamander scamander ( $821 \pm 48,50 \mathrm{mg}$ ), $\mathrm{H}$. thoas brasiliensis ( 677 $\pm 78,52 \mathrm{mg}$ ), B. polystictus polystictus ( $544 \pm 31,31 \mathrm{mg}$ ), P. anchises nephalion ( $411 \pm 13,42 \mathrm{mg}$ ) e B. polydamas polydamas (409 \pm $28,94 \mathrm{mg}$ ) apresentaram a maior massa total entre as fêmeas e P. scamander scamander $(495 \pm 25,45 \mathrm{mg}), \mathrm{H}$. thoas brasiliensis (435 $\pm 27,93 \mathrm{mg})$, B. polydamas polydamas $(329 \pm 11,49 \mathrm{mg}) \mathrm{eH}$. astyalus astyalus ( $313 \pm 11,24 \mathrm{mg}$ ), entre os machos. Os menores valores, para ambos os sexos, foram observados em $M$. Iysithous eupatorion, M. Iysithous Iysithous e M. Iysithous rurik.

Ambos os sexos de B. polydamas polydamas, H. thoas brasiliensis e $P$. scamander scamander apresentaram valores mais altos para a massa torácica média. É importante salientar que estas espécies em campo são as que mostram maior capacidade de vôo. Os menores valores, também nos dois sexos, ocorreram em $H$. hectorides, $M$. Iysithous euptorion, M. Iysithous lysithous 
Tabela I. Valores médios da massa total $(\mathrm{m})$, massa torácica $(\mathrm{mt})$ e área das asas (S) e respectivos erros padrões (ep), para ambos os sexos, de cada espécie (sp), nas comunidades estudadas (com). (n) Número de indivíduos, (Mile) Mimoides lysithous eupatorion, (M ill) M imoides lysithous lysithous, (Milr) Mimoides lysithous rurik, (Bapl) Battus polydamas polydamas, (Bapo) Battus polystictus polystictus, (Paag) Parides agavus, (Paan) Parides anchises nephalion, (Pabp) Parides bunichus perrhebus, (Heas) Heraclides astyalus astyalus, (Hehe) Heraclides hectorides, (Hetb) Heraclides thoas brasiliensis, (Ptsc) Pterourus scamander scamander, (VDRC) Vale do Diabo + Rincão do Canto, (RSML) Rincão do Soturno + Morro do Link, (AN) Água Negra, (SCCB) Schöenstatt + Camobi.

\begin{tabular}{|c|c|c|c|c|c|c|c|c|c|c|c|c|c|}
\hline \multirow[b]{2}{*}{$\mathrm{sp}$} & \multicolumn{7}{|c|}{ Machos } & \multicolumn{6}{|c|}{ Fêmeas } \\
\hline & com & $\begin{array}{c}\mathrm{m} \pm \mathrm{ep} \\
(\mathrm{mg})\end{array}$ & $\mathrm{n}$ & $\begin{array}{c}\mathrm{mt} \pm \mathrm{ep} \\
(\mathrm{mg})\end{array}$ & $n$ & $\begin{array}{l}\mathrm{S} \pm \mathrm{ep} \\
\left(\mathrm{mm}^{2}\right)\end{array}$ & $\mathrm{n}$ & $\begin{array}{c}\mathrm{m} \pm \mathrm{ep} \\
(\mathrm{mg})\end{array}$ & $\mathrm{n}$ & $\begin{array}{c}\mathrm{mt} \pm \mathrm{ep} \\
(\mathrm{mg})\end{array}$ & $\mathrm{n}$ & $\begin{array}{l}\mathrm{S} \pm \mathrm{ep} \\
\left(\mathrm{mm}^{2}\right)\end{array}$ & $\mathrm{n}$ \\
\hline \multirow[t]{5}{*}{ Mile } & VDRC & $111 \pm 10,73$ & 5 & $54 \pm 6,26$ & 5 & $1560 \pm 129,00$ & 4 & $186 \pm 54,27$ & 3 & $59 \pm 8,50$ & 4 & $1815 \pm 181,02$ & 2 \\
\hline & RSML & $144 \pm 22,50$ & 4 & $55 \pm 2,27$ & 7 & $1642 \pm 85,00$ & 4 & 0 & 0 & 0 & 0 & 1788 & 1 \\
\hline & AN & $121 \pm 7,15$ & 5 & $52 \pm 3,67$ & 6 & $1737 \pm 1,41$ & 2 & $145 \pm 12,02$ & 2 & $52 \pm 3,53$ & 2 & - & 0 \\
\hline & $\mathrm{SCCB}$ & - & 0 & - & 0 & - & 0 & 0 & 0 & 0 & 0 & - & 0 \\
\hline & Total & $123 \pm 8,02$ & 14 & $53 \pm 2,12$ & 18 & $1628 \pm 60,40$ & 10 & $169 \pm 31,30$ & 5 & $57 \pm 5,71$ & 6 & $1806 \pm 105,08$ & 3 \\
\hline \multirow[t]{5}{*}{ Mill } & VDRC & $122 \pm 7,15$ & 5 & $57 \pm 1,34$ & 5 & $1611 \pm 83,71$ & 3 & $159 \pm 28,28$ & 2 & $60 \pm 14,85$ & 2 & $1755 \pm 127,99$ & 2 \\
\hline & RSML & - & 0 & - & - & - & 0 & $199 \pm 9,19$ & 2 & $58 \pm 1,15$ & 3 & $1820 \pm 165,12$ & 3 \\
\hline & AN & $125 \pm 23,09$ & 3 & $53 \pm 1,73$ & 3 & $1534 \pm 140,71$ & 2 & - & 0 & - & 0 & - & 0 \\
\hline & $\mathrm{SCCB}$ & - & 0 & - & - & - & 0 & - & 0 & - & 0 & - & 0 \\
\hline & Total & $123 \pm 4,59$ & 8 & $55 \pm 1,06$ & 8 & $1580 \pm 66,63$ & 5 & $179 \pm 17,00$ & 4 & $59 \pm 4,92$ & 5 & $1794 \pm 100,62$ & 5 \\
\hline \multirow[t]{5}{*}{ Milr } & VDRC & $129 \pm 9,45$ & 7 & $58 \pm 2,67$ & 9 & $1643 \pm 64,91$ & 6 & $123 \pm 18,38$ & 2 & $54 \pm 1,41$ & 2 & $1525 \pm 21,92$ & 2 \\
\hline & RSML & 155 & 1 & 66 & 1 & $1823 \pm 154,86$ & 2 & $210 \pm 25,04$ & 5 & $61 \pm 6,53$ & 6 & $1789 \pm 125,74$ & 6 \\
\hline & AN & - & 0 & - & 0 & - & 0 & $181 \pm 41,01$ & 2 & $69 \pm 2,12$ & 2 & 1965 & 1 \\
\hline & $\mathrm{SCCB}$ & - & 0 & - & 0 & - & 0 & - & 0 & - & 0 & - & 0 \\
\hline & Total & $132 \pm 8,84$ & 8 & $59 \pm 2,31$ & 10 & $1688 \pm 62,93$ & 8 & $184 \pm 19,33$ & 9 & $61 \pm 4,11$ & 10 & $1750 \pm 99,35$ & 9 \\
\hline \multirow[t]{5}{*}{ Bapl } & VDRC & - & 0 & - & 0 & - & 0 & 476 & 1 & 154 & 1 & 0 & 0 \\
\hline & RSML & $340 \pm 17,95$ & 17 & $167 \pm 7,67$ & 22 & $2595 \pm 67,50$ & 16 & $409 \pm 39,19$ & 6 & $135 \pm 13,23$ & 7 & $2708 \pm 98,83$ & 5 \\
\hline & AN & $312 \pm 24,19$ & 7 & $155 \pm 8,87$ & 13 & $2452 \pm 87,18$ & 3 & $363 \pm 138,59$ & 2 & $162 \pm 23,09$ & 3 & $3111 \pm 102,53$ & 2 \\
\hline & SCCB & $320 \pm 16,26$ & 8 & $166 \pm 6,93$ & 12 & $2552 \pm 64,09$ & 6 & $445 \pm 41,72$ & 2 & $164 \pm 13,86$ & 3 & 3047 & 1 \\
\hline & Total & $329 \pm 11,49$ & 32 & $164 \pm 4,67$ & 47 & $2568 \pm 46,80$ & 25 & $409 \pm 28,94$ & 11 & $147 \pm 8,55$ & 14 & $2862 \pm 88,39$ & 8 \\
\hline \multirow[t]{5}{*}{ Bapo } & VDRC & $351 \pm 25,94$ & 5 & $140 \pm 9,80$ & 6 & $2654 \pm 170,83$ & 5 & 629 & 1 & 182 & 1 & 3418 & 1 \\
\hline & $\mathrm{RSML}$ & $277 \pm 14,72$ & 12 & $111 \pm 5,25$ & 16 & $2469 \pm 92,38$ & 12 & $503 \pm 50,09$ & 5 & $140 \pm 15,20$ & 8 & $2769 \pm 61,61$ & 7 \\
\hline & AN & $315 \pm 7,95$ & 32 & $127 \pm 2,67$ & 36 & $2522 \pm 47,54$ & 23 & $574 \pm 40,00$ & 4 & $151 \pm 6,94$ & 6 & $2851 \pm 54,27$ & 3 \\
\hline & SCCB & 309 & 1 & 132 & 1 & 2844 & 1 & - & 0 & - & 0 & - & 0 \\
\hline & Total & $309 \pm 7,21$ & 50 & $124 \pm 2,60$ & 59 & $2530 \pm 43,26$ & 41 & $544 \pm 31,31$ & 10 & $147 \pm 8,78$ & 15 & $2850 \pm 70,25$ & 11 \\
\hline \multirow[t]{5}{*}{ Paag } & VDRC & $184 \pm 5,48$ & 30 & $76 \pm 1,78$ & 38 & $1894 \pm 36,20$ & 25 & $230 \pm 34,29$ & 6 & $77 \pm 4,28$ & 14 & $2017 \pm 113,49$ & 6 \\
\hline & RSML & $194 \pm 3,67$ & 54 & $76 \pm 1,28$ & 74 & $1892 \pm 27,73$ & 59 & $259 \pm 18,86$ & 13 & $81 \pm 3,75$ & 16 & $2242 \pm 72,75$ & 16 \\
\hline & AN & $193 \pm 12,37$ & 17 & $77 \pm 2,45$ & 24 & $1957 \pm 42,60$ & 15 & 282 & 1 & $85 \pm 9,81$ & 3 & 2276 & 1 \\
\hline & SCCB & - & 0 & - & 0 & - & 0 & 311 & 1 & 98 & 1 & 0 & 0 \\
\hline & Total & $190 \pm 3,28$ & 101 & $76 \pm 0,94$ & 136 & $1902 \pm 19,90$ & 99 & $254 \pm 70,00$ & 21 & $80 \pm 2,74$ & 34 & $2185 \pm 61,09$ & 23 \\
\hline \multirow[t]{5}{*}{ Paan } & VDRC & $291 \pm 11,16$ & 27 & $118 \pm 3,12$ & 37 & $2359 \pm 60,97$ & 22 & $403 \pm 15,85$ & 23 & $123 \pm 3,85$ & 27 & $2769 \pm 63,73$ & 20 \\
\hline & RSML & $287 \pm 9,88$ & 31 & $119 \pm 3,00$ & 36 & $2344 \pm 41,41$ & 35 & $405 \pm 24,98$ & 17 & $125 \pm 4,69$ & 22 & $2738 \pm 99,66$ & 15 \\
\hline & AN & $326 \pm 32,25$ & 6 & $126 \pm 7,78$ & 8 & $2413 \pm 55,01$ & 5 & $508 \pm 16,99$ & 5 & $146 \pm 6,42$ & 7 & $2885 \pm 83,18$ & 5 \\
\hline & $\mathrm{SCCB}$ & $307 \pm 23,09$ & 3 & $125 \pm 5,20$ & 3 & $2446 \pm 12,70$ & 3 & 407 & 1 & 137 & 1 & 3071 & 1 \\
\hline & Total & $293 \pm 7,08$ & 67 & $119 \pm 1,96$ & 84 & $2359 \pm 30,51$ & 65 & $411 \pm 13,42$ & 46 & $126 \pm 2,91$ & 57 & 2779 ( 49,04 & 41 \\
\hline
\end{tabular}


Tabela I. Continuação.

\begin{tabular}{|c|c|c|c|c|c|c|c|c|c|c|c|c|c|}
\hline \multirow[b]{2}{*}{ sp } & \multicolumn{7}{|c|}{ Machos } & \multicolumn{6}{|c|}{ Fêmeas } \\
\hline & com & $\begin{array}{c}\mathrm{m} \pm \mathrm{ep} \\
(\mathrm{mg})\end{array}$ & $\mathrm{n}$ & $\begin{array}{c}\mathrm{mt} \pm \mathrm{ep} \\
(\mathrm{mg})\end{array}$ & $\mathrm{n}$ & $\begin{array}{l}\mathrm{S} \pm \mathrm{ep} \\
\left(\mathrm{mm}^{2}\right)\end{array}$ & $\mathrm{n}$ & $\begin{array}{c}\mathrm{m} \pm \mathrm{ep} \\
(\mathrm{mg})\end{array}$ & $\mathrm{n}$ & $\begin{array}{c}\mathrm{mt} \pm \mathrm{ep} \\
(\mathrm{mg})\end{array}$ & $\mathrm{n}$ & $\begin{array}{l}\mathrm{S} \pm \mathrm{ep} \\
\left(\mathrm{mm}^{2}\right)\end{array}$ & $\mathrm{n}$ \\
\hline \multirow[t]{5}{*}{ Pabp } & VDRC & $206 \pm 23,09$ & 3 & $88 \pm 7,50$ & 4 & 2247 & 1 & $302 \pm 91,92$ & 2 & $109 \pm 21,92$ & 2 & - & 0 \\
\hline & RSML & $242 \pm 6,79$ & 17 & $99 \pm 2,55$ & 26 & $2180 \pm 52,75$ & 16 & $387 \pm 19,00$ & 4 & $112 \pm 9,39$ & 5 & $2540 \pm 70,22$ & 6 \\
\hline & AN & $212 \pm 9,00$ & 16 & $91 \pm 2,34$ & 22 & $2138 \pm 53,72$ & 14 & $373 \pm 24,49$ & 6 & $115 \pm 6,33$ & 9 & $2399 \pm 50,21$ & 6 \\
\hline & SCCB & - & 0 & - & 0 & - & 0 & 0 & 0 & 0 & 0 & 0 & 0 \\
\hline & Total & $225 \pm 5,83$ & 36 & $95 \pm 1,80$ & 52 & $2163 \pm 36,10$ & 31 & $366 \pm 19,34$ & 12 & $113 \pm 5,00$ & 16 & $2469 \pm 46,19$ & 12 \\
\hline \multirow[t]{5}{*}{ Heas } & VDRC & $346 \pm 24,60$ & 5 & $142 \pm 6,05$ & 7 & $3440 \pm 119,21$ & 6 & 543 & 1 & 156 & 1 & 4033 & 1 \\
\hline & RSML & $312 \pm 12,96$ & 10 & $130 \pm 3,47$ & 14 & $3243 \pm 85,56$ & 8 & 300 & 1 & $120 \pm 26,56$ & 3 & $3678 \pm 133,64$ & 2 \\
\hline & AN & 259 & 1 & 106 & 1 & 2650 & 1 & 285 & 1 & $125 \pm 8,08$ & 3 & 3922 & 1 \\
\hline & SCCB & $282 \pm 35,22$ & 3 & $124 \pm 10,73$ & 5 & 3844 & 1 & $430 \pm 67,88$ & 2 & $141 \pm 4,24$ & 2 & $3978 \pm 2,12$ & 2 \\
\hline & Total & $313 \pm 11,24$ & 19 & $129 \pm 3,46$ & 27 & $3330 \pm 82,50$ & 16 & $398 \pm 52,32$ & 5 & $130 \pm 9,54$ & 8 & $3878 \pm 73,48$ & 6 \\
\hline \multirow[t]{5}{*}{ Hehe } & VDRC & $128 \pm 10,50$ & 4 & $63 \pm 6,26$ & 5 & $1714 \pm 183,14$ & 2 & 0 & 0 & 48 & 1 & 0 & 0 \\
\hline & RSML & $173 \pm 7,56$ & 7 & $76 \pm 1,94$ & 13 & $2286 \pm 44,09$ & 6 & $243 \pm 37,12$ & 5 & $69 \pm 5,71$ & 6 & $2186 \pm 91,68$ & 5 \\
\hline & AN & $154 \pm 16,50$ & 4 & $67 \pm 5,37$ & 5 & $2168 \pm 149,00$ & 4 & 0 & 0 & 85 & 1 & 0 & 0 \\
\hline & SCCB & - & 0 & - & 0 & - & 0 & 296 & 1 & 99 & 1 & 2812 & 1 \\
\hline & Total & $156 \pm 7,75$ & 15 & $71 \pm 2,29$ & 23 & $2151 \pm 81,69$ & 12 & $239 \pm 31,84$ & 6 & $71 \pm 6,01$ & 8 & $2302 \pm 117,98$ & 6 \\
\hline \multirow[t]{5}{*}{ Hetb } & VDRC & 574 & 1 & 232 & 1 & 4645 & 1 & - & 0 & - & 0 & - & 0 \\
\hline & $\mathrm{RSML}$ & $424 \pm 19,05$ & 3 & $185 \pm 6,00$ & 4 & $4098 \pm 229,81$ & 2 & - & 0 & 130 & 1 & - & 0 \\
\hline & AN & $452 \pm 74,25$ & 2 & $198 \pm 24,04$ & 2 & 4745 & 1 & 498 & 1 & 175 & 1 & - & 0 \\
\hline & SCCB & $364 \pm 4,24$ & 2 & $167 \pm 11,02$ & 6 & $4287 \pm 223,44$ & 2 & $729 \pm 79,20$ & 2 & $199 \pm 26,87$ & 2 & 4473 & 1 \\
\hline & Total & $435 \pm 27,93$ & 8 & $183 \pm 7,76$ & 13 & $4359 \pm 139,21$ & 6 & $677 \pm 78,52$ & 3 & $168 \pm 21,94$ & 3 & 4473 & 1 \\
\hline \multirow[t]{5}{*}{ Ptsc } & VDRC & - & 0 & - & 0 & - & 0 & - & 0 & - & 0 & - & 0 \\
\hline & $\mathrm{RSML}$ & 582 & 1 & 227 & 1 & - & 0 & - & 0 & - & 0 & - & 0 \\
\hline & AN & - & 0 & - & 0 & - & 0 & - & 0 & - & 0 & - & 0 \\
\hline & $\mathrm{SCCB}$ & $482 \pm 25,32$ & 7 & $205 \pm 8,66$ & 12 & 3869 & 1 & $821 \pm 48,50$ & 4 & $235 \pm 11,46$ & 11 & $3870 \pm 108,54$ & 3 \\
\hline & Total & $495 \pm 25,45$ & 8 & $207 \pm 8,04$ & 13 & 3869 & 1 & $821 \pm 48,50$ & 4 & $235 \pm 11,46$ & 11 & $3870 \pm 108,54$ & 3 \\
\hline
\end{tabular}

e M. Iysithous rurik. A correlação entre a massa total e a massa torácica foi significativamente alta em ambos os sexos (machos: $r=0,95 ; r^{2}=0,91 ; n=377 ; p<0,01$; fêmeas: $r=0,95 ; r^{2}=$ $0,91 ; n=150 ; p<0,01)$.

MARDEN \& ChAl (1991) sugerem que borboletas palatáveis podem apresentar uma proporção maior na musculatura de vôo por massa corporal, que pode ser usada para estabelecer uma estratégia importante no vôo evasivo. Por isso voam rápido, ao contrário de espécies impalatáveis que são mais lentas e apresentam asas mais resistentes (PINHeIRo 1996). Neste trabaIho, as espécies com maior massa total foram aquelas citadas na literatura, normal mente, como palatáveis. Pterourus scamander scamander e H. astyalus astyalus são considerados mímicos que têm espécies de Battus como modelo (TYLER et al. 1994). Na Costa Rica Callocitta formosa (Passeriformes; Corvidae) recusa sistematicamente exemplares de Battus spp. (DEVRIES 1986). Contudo, testes de palatabilidade com Battus polydamas mostraram que $33 \%$ dos indivíduos oferecidos foram comidos (SRYGley \& Chal 1990). Heraclides hectorides, M. Iysithous eupatorion, M. Iysithous lysithous, M. Iysithous rurik, P. anchises nephalion e $P$. bunichus perrhebus são apresentados como comímicos do padrão "Parides", cujas espécies apresentam uma rejeição de 70-80\% (PInHeIro 1996) e $\mathrm{H}$. thoas brasiliensis tida como palatável foi atacada em $88 \%$ dos testes (SRYGLEY \& CHAI 1990). Por outro lado algumas espécies como Heraclides anchisiades eProtesilaus sp. embora citadas como palatáveis são rejeitadas (Tyler et al. 1994). Segundo RACHel \& PARISET (1992) as fêmeas de Heraclides androgeus, mímicas de Battus, são rapidamente atacadas e comidas, mas as de Heraclides anchisiades, que faz parte de outro anel mimético com fêmeas de várias 
espécies de Parides, não são atacadas, embora em SRYGLEY \& CHAI (1990) sejam citadas como mímicos batesianos. É importante salientar que os dados sobre a palatabilidade das espécies citadas foram obtidos em locais distantes e com diferentes aves testes, o que poderia suscitar dúvidas quanto ao grau de palatabilidade (SRYGLEY \& Chal 1990). Por conveniência, foi assumida uma equivalência na palatabilidade, como no trabalho de BRower \& BRower (1964). Nesses estudos, os resultados sobre a palatabilidade foram qualitativamente semelhantes, usando borboletas da mesma espécie, provenientes de locais distantes, e utilizando diferentes espécies de aves como predadores. Além disso, ao nível de comunidade pode ser assumido que as pressões sel etivas também são semelhantes (SRYGLEY \& CHAI 1990).

Ambos os sexos deH. astyalus astyalus, $H$. thoas brasiliensis e P. scamander scamander, que ocupam preferencialmente locais abertos e real izam deslocamentos a longa distância, exibiram os valores mais al tos para a área das asas. M imoides lysithous eupatorion, M. Iysithous lysithous, M. Iysithous rurik, e P. agavus apresentaram os menores valores e todas são encontradas geralmenteem matas eflorestas. É importante sal ientar que quanto maior o tamanho das asas, maior o peso que elas podem carregar. Deste modo, os deslocamentos a longa distância necessitam uma massa muscular maior.

Os val ores obti dos mostram que a massa torácica, o comprimento e a área das asas estão todos intimamente relacionados em Papilioninae, eque os aumentos nestes três parâmetros são diretamente proporcionais. Os resultados indicam também que as proporções se ajustam a massa final do corpo. A correlação entre a massa torácica $\left(\mathrm{m}_{\mathrm{t}}\right)$ e a área das asas (S) (machos: $\mathrm{r}$ $=0,83 ; r^{2}=0,69 ; n=290 ; \mathrm{F}=658,0 ; \mathrm{gl}=1,288 ; \mathrm{p}<0.001$; fêmeas: $r=0,80 ; r^{2}=0,65 ; n=112 ; F=200,48 ; g l=1,110 ; p<$ $0,001)$, entre a envergadura da asa $(2 \mathrm{C})$ e a massa torácica $\left(\mathrm{m}_{\mathrm{t}}\right)$ (machos: $r=0,80 ; r^{2}=0,65 ; n=462 ; F=845,88 ; g l=1,460 ; p<$ 0,001; fêmeas: $r=0,77 ; r^{2}=0,59 ; n=183 ; F=259,67 ; g l=1$, $181 ; p<0,001$ ) e entre a envergadura (2C) e a área das asas (S) (machos: $r=0,97 ; r^{2}=0,93 ; n=322 ; F=4505,83 ; g l=1,320 ; p$ $<0,001$; fêmeas: $r=0,96 ; r^{2}=0,92 ; n=132 ; F=1663,11 ; g l=1$, 130; $p<0,001$ ) foram altamente significativas e não diferem estatisticamente entre os sexos.

Uma comparação simultânea com as espécies agrupadas por sexo e por localidade, para a massa torácica (Tab. II), área das asas (Tab. II) e envergadura das asas (Tab. II), através dos testes LSD e Bonferroni, mostra que as diferenças entre os valores médios de SCCB e as demais comunidades foram estatisticamente significantes. À medida que a cobertura vegetal vai diminuindo, os valores médios vão se elevando, exceto para a área das asas das fêmeas, onde RSML apresentou um valor menor queVDRC, embora a diferença não seja significante. A diferença entre AN eVDRC é significativa, somente com o teste LSD, para a massa torácica e envergadura das asas, em ambos os sexos.

Em ambientes menos favorecidos a sel eção pode ben eficiar machos com maior massa muscular. Em Scathophaga stercoraria (Linnaeus, 1758) (Diptera, Scathophagidae), por exemplo, fêmeas que acasalam com machos menores têm menor probabilidade de sobrevivência durante a conjugação da cópula (BoRGIA 1981), pois estariam mais vulneráveis a predação já que são os machos que carregam as fêmeas ao se deslocarem durante a cópula. Portanto, borboletas com tamanhos maiores em locais com pouca vegetação ou condições menos adequadas, também, poderiam obter uma vantagem adaptativa, aumentando a sua performance de vôo e chances reprodutivas nestes locais, quando associadas a uma dinâmica de vôo que favorece a sobrevivência e a reprodução.

Através de estudos adequados, as diferenças observadas entre machos e fêmeas poderiam ser atribuídas a uma divergência intersexual de nicho ou a um dimorfismo sexual ecológico. Estas diferenças, observadas na morfologia associada ao vôo, poderiam influenciar a habilidade das borboletas colonizarem hábitats fragmentados, como AN e SCCB. O polimorfismo de asas é comum em muitas ordens de insetos, e a perda ou redução das asas é geralmente associada com aumento na fecundidade e uma precocidade nas formas imaturas, dando desta maneira uma vantagem reprodutiva as formas não migrantes (HARRISON 1980, RoFf 1986).

As médias do comprimento (C) e da largura (L) das asas anteriores (a) e posteriores ( $p$ ) (Tab. III), foram estatisticamente diferentes entre os sexos (Ca: $\mathrm{t}_{(726)}=6,568 ; \mathrm{p}<0,01$; La: $\mathrm{t}_{(733)}=$ 10,$952 ; \mathrm{p}<0,01 ; \mathrm{Cp}: \mathrm{t}_{(587)}=2,974 ; \mathrm{p}<0,01 ; \mathrm{Lp:} \mathrm{t}_{(587)}=7,943 ; \mathrm{p}<$ $0,01)$. Os valores mais baixos, em ambos os sexos, para o comprimento e a largura das asas anteriores, nas quatro comunidades estudadas ocorreram em M. Iysithous eupatorion, M. Iysithous Iysithous, M. Iysithous rurik, e P. agavus. Os valores mais baixos para a largura média das asas anteriores, em ambos os sexos, foram evidenciados nas três subespécies de Mimoides e em $P$. agavus. A largura das asas posteriores, nos machos, apresentou valores menores em $\mathrm{H}$. hectorides, M. Iysithous eupatorion, $M$. Iysithous lysithous, M. Iysithous rurik, P. agavus eP. anchises nephalion, enquanto nas fêmeas somente nas subespécies de Mimoides. A espécie que apresentou os valores médios mais al tos para as duas variáveis, em ambas as asas e sexos, foi $\mathrm{H}$. thoas brasiliensis.

Na tabela IV são analisadas as correlações entre o comprimento e a largura, por sexo e por asa. Tanto para os machos como para as fêmeas, as correlações que incluem o comprimento das asas anteriores ( $\mathrm{Ca}$ ) foram todas positivas e altamente significantes não diferindo entre os sexos. Já as que incluem o comprimento das asas posteriores (Cp) foram baixase diferentes entre os sexos. Deste modo, com base nestes resultados podemos inferir que o comprimento das asas anteriores, pela sua semel hança entre os sexos e grau de correl ação, é uma medida alométrica importante na determinação do tamanho e forma das asas. Tal fato pode ser atribuído ao papel que as asas anteriores desempenham na aerodinâmica e sua relação com a velocidade. Já nas asas posteriores, a maior variabilidade e a diferença observada entre os sexos podem ser atribuídas ao papel que elas exercem, funcionando como um aerofólio, regulando a direção e a manobrabilidade do vôo. 
Tabela II. Comparação simultânea dos valores médios obtidos $(\mathrm{x})$, nas diferentes comunidades (com) estudadas, para a: (A) massa torácica (mg), (B) área das asas ( $\mathrm{mm} 2$ ) e (C) envergadura das asas ( $\mathrm{mm})$, através dos testes LSD e Bonferroni (Bonf), para ambos os sexos, nas comunidades estudadas. Valores médios seguidos pela mesma letra, em cada coluna, não diferem estatisticamente $(p>0,05)$. (VDRC) Vale do Diabo + Rincão do Canto, (RSML) Rincão do Soturno + Morro do Link, (AN) Água Negra, (SCCB) Schöenstatt + Camobi.

\begin{tabular}{|c|c|c|c|c|c|c|c|c|c|c|c|c|c|c|c|c|c|c|}
\hline \multirow{3}{*}{ Com } & \multicolumn{6}{|c|}{ Massa torácica $(\mathrm{mg})$} & \multicolumn{6}{|c|}{ Área das asas $\left(\mathrm{mm}^{2}\right)$} & \multicolumn{6}{|c|}{ Envergadura das asas (mm) } \\
\hline & \multicolumn{3}{|c|}{ Machos } & \multicolumn{3}{|c|}{ Fêmeas } & \multicolumn{3}{|c|}{ Machos } & \multicolumn{3}{|c|}{ Fêmeas } & \multicolumn{3}{|c|}{ Machos } & \multicolumn{3}{|c|}{ Fêmeas } \\
\hline & $x$ & LSD & Bonf & $x$ & LSD & Bonf & $x$ & LSD & Bonf & $x$ & LSD & Bonf & $x$ & LSD & Bonf & $x$ & LSD & Bonf \\
\hline VDRC & 118,93 & a & a & 127,71 & a & a & 2199 & a & a & 2504 & a & a & 73,88 & a & a & 78,18 & a & a \\
\hline RSML & 129,56 & b & a & 131,96 & a & a & 2236 & a & a & 2445 & a & a & 75,12 & $a, b$ & a & 80,06 & a & a \\
\hline AN & 133,91 & $b$ & a & 154,18 & $b$ & a & 2278 & a & a & 2724 & a & a & 76,65 & $b$ & a & 84,48 & b & a \\
\hline SCCB & 209,31 & $C$ & $b$ & 231,39 & C & b & 2984 & $b$ & $b$ & 3663 & $b$ & $b$ & 92,08 & C & $b$ & 96,42 & C & b \\
\hline
\end{tabular}

Deste modo as asas posteriores podem estar relacionadas ao tipo de vôo que as borboletas exercem (DudLEY 2000). Segundo BRodSKY (1994) os tamanhos do corpo e das asas predispuseram os lepidópteros ao domínio do vôo planado, especialmente os papilionídeos, pela presença de caudas nas asas posteriores, que são responsáveis pela sua orientação e direção. Nestetipo de vôo, o perfil geral da trajetória éimprevisível e complicada. Deste modo, a variabilidade observada nas asas posteriores pode ser atribuída à seleção de comportamentos especificamente associados ao vôo destas borboletas.

\section{Medidas aerodinâmicas}

Os valores obtidos para a carga unitária $(\mathrm{Cu})$, índice de estreiteza (E) e velocidade estimada com base no índice de estreiteza $\left(\mathrm{V}_{\mathrm{E}}\right)$, para ambos os sexos, nas diferentes comunidades estudadas podem ser visualizados na tabela V. As estimativas médias mais baixas para a carga unitária foram observadas em Mimoides sspp. eH. hectorides, para ambos os sexos. As mais elevadas em $B$. polydamas polydamas, B. polystictus polystictus e $P$. anchises nephalion, para os machos, eB. polydamas polydamas, B. polystictus polystictus, $P$. anchises nephalion eP. bunichus perrhebus, para as fêmeas. Para o índice de estreiteza, os menores valores foram verificados em $P$. agavus, $P$. anchises nephalion eP. bunichus perrhebus, e os maiores em $\mathrm{B}$. polystictus polystictus, $\mathrm{H}$. astyalus astyalus, $\mathrm{H}$. hectorides eH. thoas brasiliensis, nos dois sexos. Também, para machos e fêmeas, os menores val ores para a velocidade ocorreram em B. polystictus polystictus, $\mathrm{H}$. astyalus astyalus, $\mathrm{H}$. hectorides e $\mathrm{H}$. thoas brasiliensis, e os maiores em P. agavus, $\mathrm{P}$. anchises nephalion e P. bunichus perrhebus. A distribuição dos valores médios (Fig. 2), entre os sexos, das estimativas do índice de estreiteza e da velocidade estimada com basenesteíndice, quando analisadas através de teste-t, não foram estatisticamente significativas $\left(\mathrm{t}_{(446)}=0,718 ; \mathrm{p}>0,05 ; \mathrm{t}_{(446)}=-0,718 ; \mathrm{p}>0,05\right.$, respectivamente). Entretanto, foi significativa em relação a carga unitária $\left(\mathrm{t}_{(353)}=10,183 ; \mathrm{p}<0,05\right)$ (Fig. 3). Os resultados, embora semel hantes, são mais baixos que os obtidos por DudLeY \& SRYGLEY (1994) para a subfamília Papilioninae ( $\mathrm{m}=416 \mathrm{mg}$; $=3,39 ; \mathrm{Cu}=1,45$ $\mathrm{mg} / \mathrm{mm}^{2} ; \mathrm{V}=4,4 \mathrm{~m} / \mathrm{s}$ ) ou individualmente para a espécie $B$. polydamas ( $\mathrm{m}=551 \mathrm{mg} ; \mathrm{E}=3,54 ; \mathrm{Cu}=1,89 \mathrm{mg} / \mathrm{mm}^{2} ; \mathrm{V}=5,8 \mathrm{~m} / \mathrm{s}$ ).
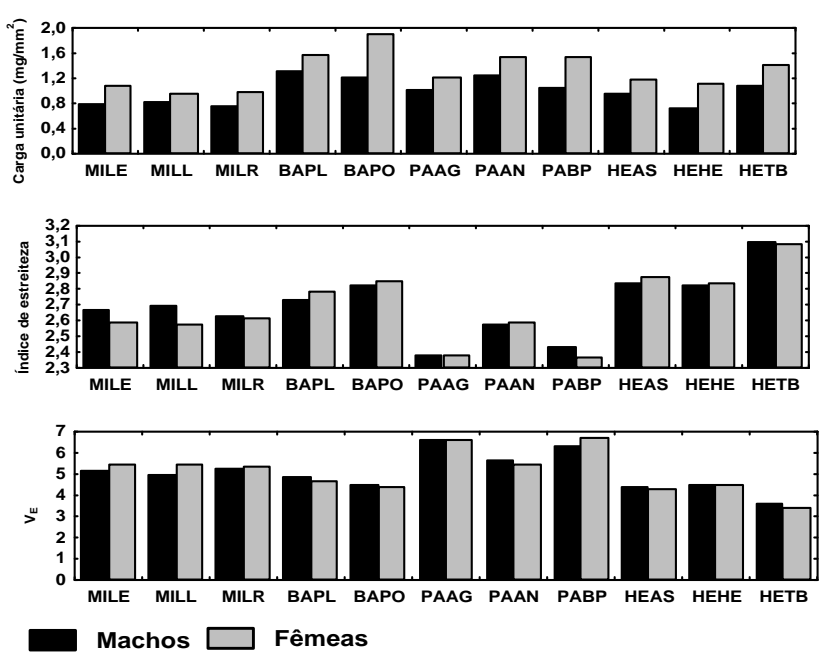

Figura 2. Distribuição dos valores médios da carga unitária, do índice de estreiteza e da previsão da velocidade com base no índice de estreiteza $\left(\mathrm{V}_{\mathrm{E}}\right)$, por sexo e por espécie: (BAPL) Battus polydamas, (BAPO) Battus polystictus, (HEAS) Heraclides astyalus astyalus, (HEHE) Heraclides hectorides, (HETB) Heraclides thoas brasiliensis, (MILE) Mimoides lysithous eupatorion, (MILL) M imoides lysithous lysithous, (MILR) Mimoides lysithous rurik, (PAAG) Parides agavus, (PAAN) Parides anchises nephalion, (PABP) Parides bunichus perrhebus.

A habilidade de fuga das borboletas é a principal forma de evitar predadores. Borboletas impalatáveis apresentam índice de estreiteza el evado e os palatáveisíndices baixos (DUDLEY 2000). Estudos que relacionam a forma das asas com palatabilidade demonstram que as borboletas impalatáveis apresentam asas anteriores longas e estreitas, enquanto as palatáveis asas curtas e largas (CHAI \& SRYGLEY 1990). Asas Iongas e estreitas permitem um vôo planado enquanto asas curtas e largas uma manobrabilidade maior (DUDLEY 2000). Todas as borboletas, mesmo as impalatáveis, respondem aos predadores com padrões de vôo em ziguezague e um movimento para 


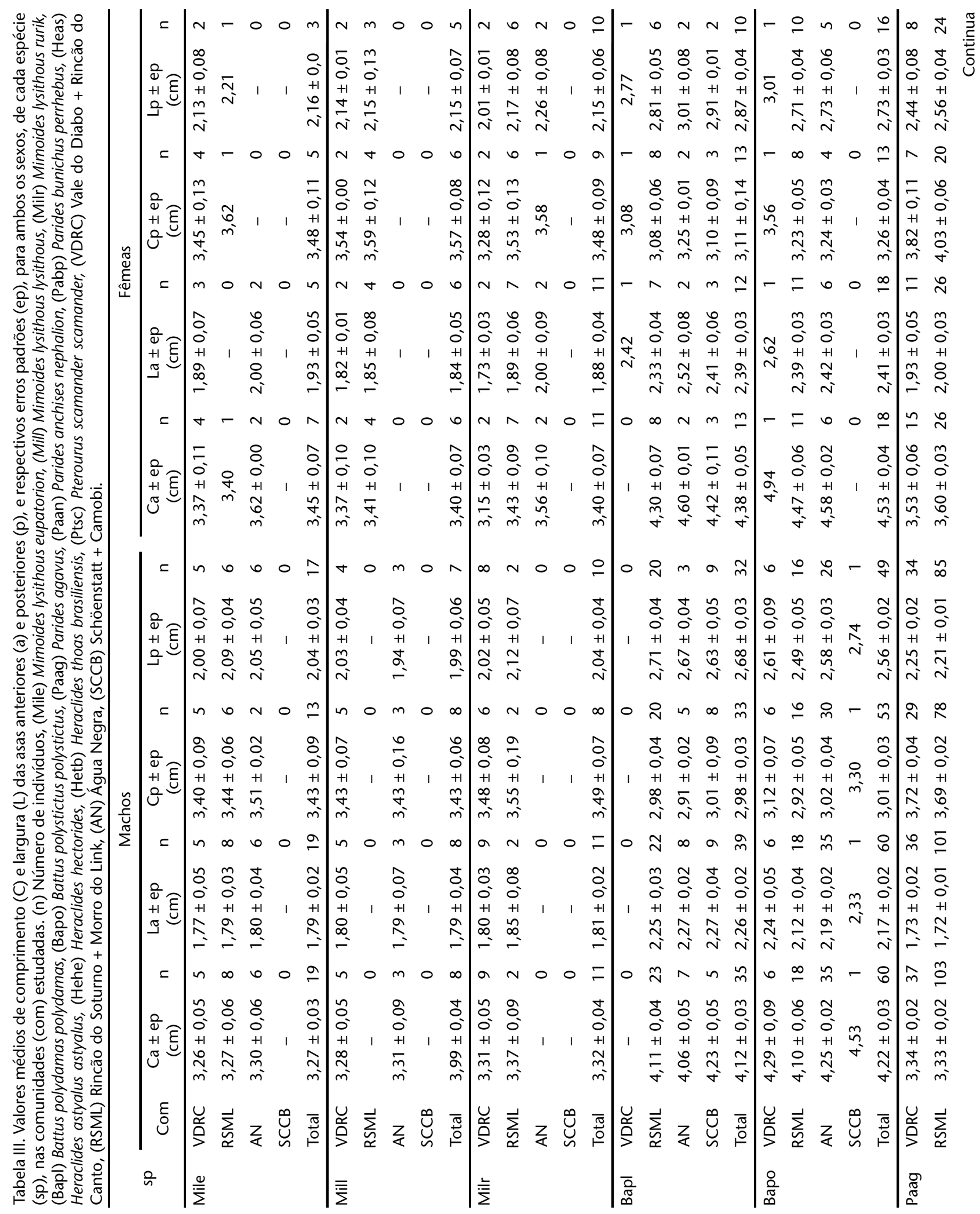

Revista Brasileira de Zoologia 21 (4): 833-846, dezembro 200W 


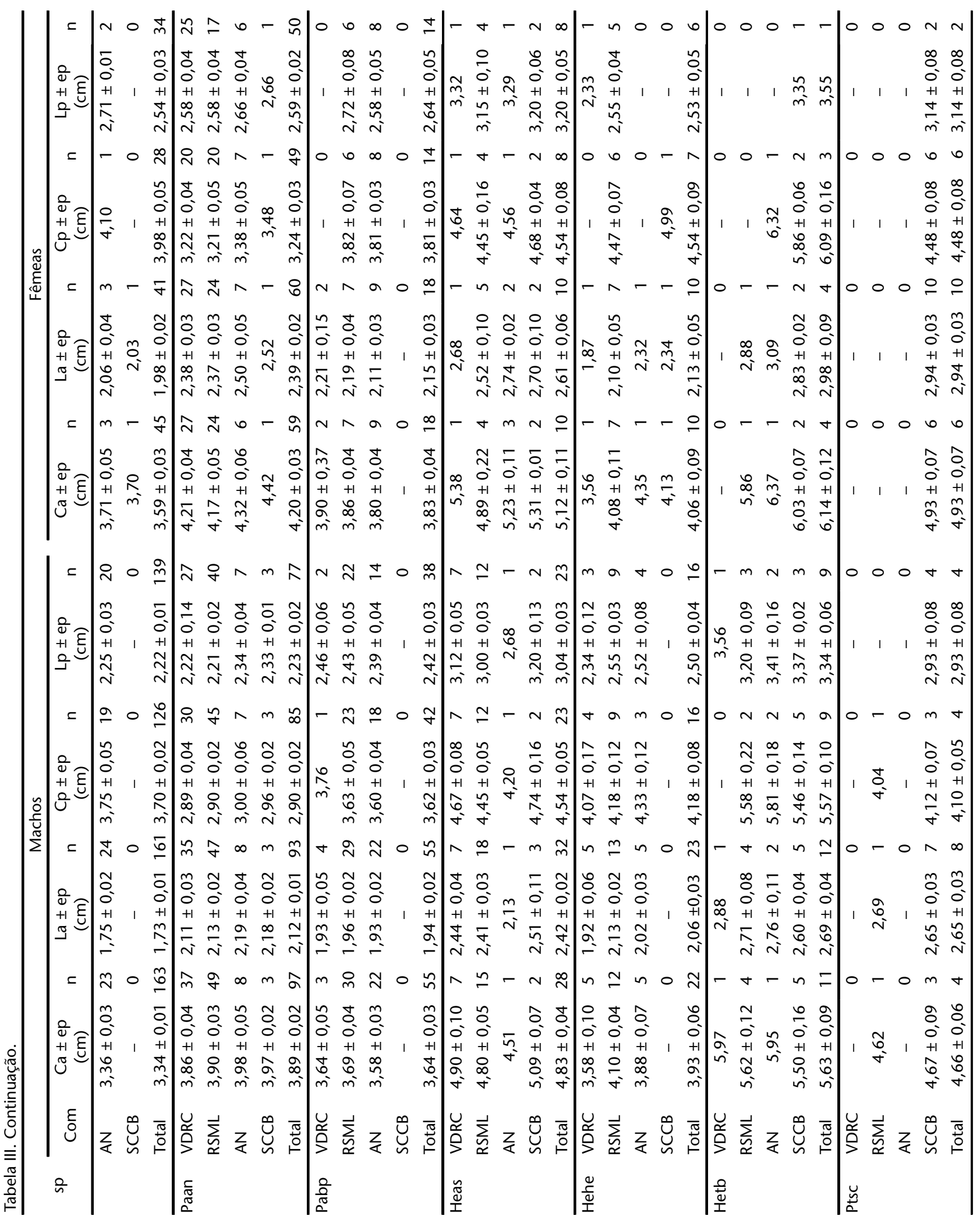

Revista Brasileira de Zoologia 21 (4): 833-846, dezembro 200W 


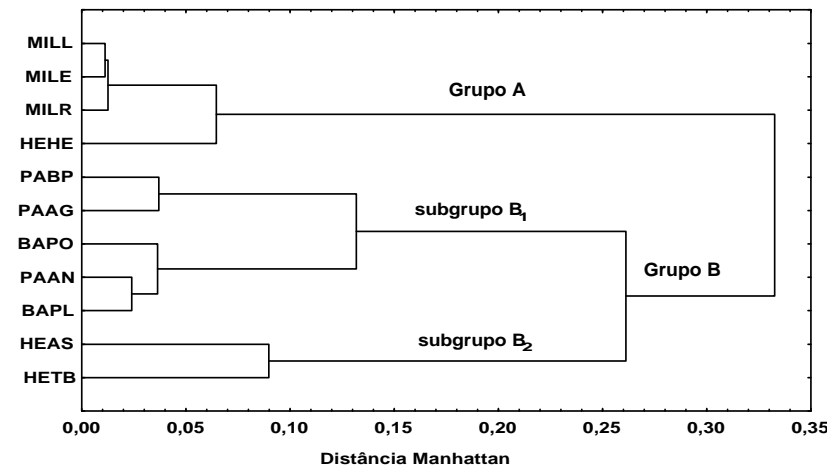

Figura 3. Agrupamento das espécies estudadas com base no índice de estreiteza, carga unitária e área alar das asas anteriores, utilizando método Ward e distância de Manhattan.
Tabela IV. Correlações entre o comprimento (C) e a largura (L) das asas anteriores (a) e posteriores ( $p$ ) de machos e fêmeas coletados nas diferentes comunidades estudadas. ( $r$ ) Coeficiente de correlação, $\left(r^{2}\right)$ coeficiente de determinação, (C) comprimento, (L) largura, (a) anterior, (p) posterior, $(*) p<0,05$, (NS) não significante.

\begin{tabular}{cccccccc}
\hline & \multicolumn{2}{c}{ Machos } & \multicolumn{4}{c}{ Fêmeas } \\
\hline Cax La & r & r2 & & r & r2 & \\
\hline Cax Cp & 0,15 & 0,89 & $*$ & 0,94 & 0,89 & $*$ \\
Ca X Lp & 0,87 & 0,75 & $*$ & 0,84 & 0,71 & $*$ \\
Cp x Lp & 0,43 & 0,18 & $*$ & 0,43 & 0,18 & $*$ \\
La X Lp & 0,81 & 0,65 & $*$ & 0,82 & 0,66 & $*$ \\
Cp x La & 0,09 & 0,009 & NS & 0,28 & 0,08 & $*$ \\
\hline
\end{tabular}

Tabela V. Valores médios da carga unitária (Cu), do índice de estreiteza (E) e da velocidade estimada com base no índice de estreiteza (VE), e respectivos erros padrões (ep), de ambos os sexos, nas quatro comunidades (com) investigadas. (n) Número de indivíduos, (Mile) M imoides lysithous eupatorion, (M ill) M imoides lysithous lysithous, (Milr) M imoides lysithous rurik, (Bapl) Battus polydamas polydamas, (Bapo) Battus polystictus polystictus, (Paag) Parides agavus, (Paan) Parides anchises nephalion, (Pabp) Parides bunichus perrhebus, (Heas) Heraclides astyalus astyalus, (Hehe) Heraclides hectorides, (Hetb) Heraclides thoas brasiliensis, (Ptsc) Pterourus scamander scamander, (VDRC) Vale do Diabo + Rincão do Canto, (RSML) Rincão do Soturno + Morro do Link, (AN) Água Negra, (SCCB) Schöenstatt + Camobi.

\begin{tabular}{|c|c|c|c|c|c|c|c|c|c|c|c|c|c|}
\hline \multirow[b]{2}{*}{$\mathrm{sp}$} & \multirow[b]{2}{*}{ com } & \multicolumn{6}{|c|}{ Machos } & \multicolumn{6}{|c|}{ Fêmeas } \\
\hline & & $\begin{array}{c}\mathrm{Cu} \pm \mathrm{ep} \\
\left(\mathrm{mg} / \mathrm{mm}^{2}\right)\end{array}$ & $\mathrm{n}$ & $E \pm$ ep & $\mathrm{n}$ & $\begin{array}{c}\text { VE } \pm \text { ep } \\
(\mathrm{m} / \mathrm{s})\end{array}$ & $\mathrm{n}$ & $\begin{array}{c}\mathrm{Cu} \pm \mathrm{ep} \\
\left(\mathrm{mg} / \mathrm{mm}^{2}\right)\end{array}$ & $\mathrm{n}$ & $E \pm$ ep & $\mathrm{n}$ & $\begin{array}{c}\text { VE } \pm \text { ep } \\
(\mathrm{m} / \mathrm{s})\end{array}$ & $\mathrm{n}$ \\
\hline \multirow[t]{5}{*}{ Mile } & VDRC & $0,77 \pm 0,01$ & 4 & $2,75 \pm 0,13$ & 4 & $4,84 \pm 0,46$ & 4 & $1,09 \pm 0,37$ & 2 & $2,58 \pm 0,05$ & 2 & $5,46 \pm 0,35$ & 2 \\
\hline & RSML & $0,86 \pm 0,08$ & 4 & $2,66 \pm 0,04$ & 4 & $5,12 \pm 0,19$ & 4 & - & 0 & 2,60 & 1 & 5,36 & 1 \\
\hline & AN & $0,71 \pm 0,06$ & 2 & $2,53 \pm 0,05$ & 2 & $5,72 \pm 0,25$ & 2 & - & 0 & - & 0 & - & 0 \\
\hline & SCCB & - & 0 & - & 0 & & 0 & - & 0 & - & 0 & - & 0 \\
\hline & Total & $0,79 \pm 0,04$ & 10 & $2,67 \pm 0,06$ & 10 & $5,13 \pm 0,21$ & 10 & $1,09 \pm 0,37$ & 2 & $2,59 \pm 0,02$ & 3 & $5,42 \pm 0,20$ & 3 \\
\hline \multirow[t]{5}{*}{ Mill } & VDRC & $0,81 \pm 0,11$ & 3 & $2,63 \pm 0,03$ & 3 & $5,24 \pm 0,13$ & 3 & $0,90 \pm 0,10$ & 2 & $2,60 \pm 0,02$ & 2 & $5,39 \pm 0,16$ & 2 \\
\hline & RSML & - & 0 & - & 0 & - & 0 & $1,01 \pm 0,06$ & 2 & $2,57 \pm 0,02$ & 3 & $5,52 \pm 0,27$ & 3 \\
\hline & AN & $0,82 \pm 0,05$ & 2 & $2,79 \pm 0,03$ & 2 & $4,55 \pm 0,10$ & 2 & - & 0 & & 0 & - & 0 \\
\hline & SCCB & - & 0 & - & 0 & - & 0 & - & 0 & & 0 & - & 0 \\
\hline & Total & $0,81 \pm 0,06$ & 5 & $2,69 \pm 0,04$ & 5 & $4,96 \pm 0,18$ & 5 & $0,95 \pm 0,05$ & 4 & $2,58 \pm 0,01$ & 5 & $5,46 \pm 0,16$ & 5 \\
\hline \multirow[t]{5}{*}{ Milr } & VDRC & $0,76 \pm 0,04$ & 6 & $2,66 \pm 0,04$ & 6 & $5,13 \pm 0,17$ & 6 & $0,80 \pm 0,11$ & 2 & $2,61 \pm 0,01$ & 2 & $5,31 \pm 0,07$ & 2 \\
\hline & $\mathrm{RSML}$ & 0,78 & 1 & $2,51 \pm 0,07$ & 2 & $5,82 \pm 0,38$ & 2 & $1,13 \pm 0,07$ & 5 & $2,64 \pm 0,03$ & 6 & $5,20 \pm 0,15$ & 6 \\
\hline & AN & - & 0 & - & 0 & - & 0 & 0,71 & 1 & 2,45 & 1 & 6,15 & 1 \\
\hline & SCCB & - & 0 & - & 0 & - & 0 & - & 0 & - & 0 & - & 0 \\
\hline & Total & $0,76 \pm 003$ & 7 & $2,62 \pm 0,04$ & 8 & $5,30 \pm 0,18$ & 8 & $1,00 \pm 0,08$ & 8 & $2,61 \pm 0,09$ & 9 & $5,33 \pm 0,14$ & 9 \\
\hline \multirow[t]{5}{*}{ Bapl } & VDRC & - & 0 & - & 0 & - & 0 & - & 0 & - & 0 & - & 0 \\
\hline & RSML & $1,32 \pm 0,06$ & 12 & $2,70 \pm 0,02$ & 16 & $4,95 \pm 0,09$ & 16 & $1,65 \pm 0,10$ & 4 & $2,81 \pm 0,12$ & 5 & $4,52 \pm 0,19$ & 5 \\
\hline & AN & $1,18 \pm 0,06$ & 3 & $2,73 \pm 0,02$ & 3 & $4,79 \pm 0,09$ & 3 & 1,67 & 1 & $2,72 \pm 0,09$ & 2 & $4,83 \pm 0,27$ & 2 \\
\hline & SCCB & $1,33 \pm 0,06$ & 6 & $2,82 \pm 0,03$ & 5 & $4,46 \pm 0,09$ & 5 & 1,60 & 1 & 2,80 & 1 & 4,52 & 1 \\
\hline & Total & $1,30 \pm 0,04$ & 21 & $2,73 \pm 0,02$ & 24 & $4,83 \pm 0,07$ & 24 & $1,59 \pm 0,08$ & 6 & $2,78 \pm 0,10$ & 8 & $4,63 \pm 0,13$ & 8 \\
\hline \multirow[t]{3}{*}{ Bapo } & VDRC & $1,32 \pm 0,05$ & 4 & $2,80 \pm 0,05$ & 5 & $4,55 \pm 0,17$ & 5 & 1,84 & 1 & 2,86 & 1 & 4,31 & 1 \\
\hline & $\mathrm{RSML}$ & $1,13 \pm 0,04$ & 9 & $2,76 \pm 0,03$ & 12 & $4,68 \pm 0,10$ & 12 & $1,82 \pm 0,26$ & 3 & $2,81 \pm 0,05$ & 7 & $4,48 \pm 0,06$ & 7 \\
\hline & AN & $1,25 \pm 0,02$ & 23 & $2,86 \pm 0,03$ & 23 & $4,37 \pm 0,09$ & 23 & $1,98 \pm 0,18$ & 3 & $2,92 \pm 0,06$ & 3 & $4,12 \pm 0,10$ & 3 \\
\hline
\end{tabular}

Continua 
Tabela V. Continuação.

\begin{tabular}{|c|c|c|c|c|c|c|c|c|c|c|c|c|c|}
\hline \multirow[b]{2}{*}{$\mathrm{sp}$} & \multirow[b]{2}{*}{ com } & \multicolumn{6}{|c|}{ Machos } & \multicolumn{6}{|c|}{ Fêmeas } \\
\hline & & $\begin{array}{c}\mathrm{Cu} \pm \mathrm{ep} \\
\left(\mathrm{mg} / \mathrm{mm}^{2}\right)\end{array}$ & $\mathrm{n}$ & $E \pm$ ep & $\mathrm{n}$ & $\begin{array}{c}\text { VE } \pm \text { ep } \\
(\mathrm{m} / \mathrm{s})\end{array}$ & $\mathrm{n}$ & $\begin{array}{c}\mathrm{Cu} \pm \mathrm{ep} \\
\left(\mathrm{mg} / \mathrm{mm}^{2}\right)\end{array}$ & $\mathrm{n}$ & $E \pm$ ep & $\mathrm{n}$ & $\begin{array}{c}\text { VE } \pm \text { ep } \\
(\mathrm{m} / \mathrm{s})\end{array}$ & $\mathrm{n}$ \\
\hline & SCCB & 1,09 & 1 & 2,89 & 1 & 4,23 & 1 & - & 0 & - & 0 & - & 0 \\
\hline & Total & $1,22 \pm 0,02$ & 37 & $2,82 \pm 0,02$ & 41 & $4,48 \pm 0,07$ & 41 & $1,89 \pm 0,12$ & 7 & $2,85 \pm 0,07$ & 11 & $4,37 \pm 0,07$ & 11 \\
\hline \multirow[t]{5}{*}{ Paag } & VDRC & $0,98 \pm 0,02$ & 24 & $2,39 \pm 0,02$ & 25 & $6,53 \pm 0,09$ & 25 & $1,18 \pm 0,06$ & 4 & $2,47 \pm 0,21$ & 6 & $6,14 \pm 0,43$ & 6 \\
\hline & RSML & $1,02 \pm 0,01$ & 37 & $2,37 \pm 0,01$ & 59 & $6,62 \pm 0,08$ & 59 & $1,20 \pm 0,07$ & 9 & $2,35 \pm 0,09$ & 16 & $6,77 \pm 0,15$ & 16 \\
\hline & AN & $1,05 \pm 0,06$ & 12 & $2,39 \pm 0,03$ & 15 & $6,54 \pm 0,18$ & 15 & $1,24 \pm 0,00$ & 1 & 2,38 & 1 & 6,58 & 1 \\
\hline & $\mathrm{SCCB}$ & - & 0 & - & 0 & - & 0 & - & 0 & - & 0 & - & 0 \\
\hline & Total & $1,01 \pm 0,01$ & 73 & $2,38 \pm 0,01$ & 99 & $6,59 \pm 0,06$ & 99 & $1,20 \pm 0,04$ & 14 & $2,38 \pm 0,14$ & 23 & $6,60 \pm 0,16$ & 23 \\
\hline \multirow[t]{5}{*}{ Paan } & VDRC & $1,26 \pm 0,05$ & 18 & $2,54 \pm 0,05$ & 22 & $5,87 \pm 0,40$ & 22 & $1,51 \pm 0,04$ & 18 & $2,59 \pm 0,08$ & 20 & $5,43 \pm 0,09$ & 20 \\
\hline & RSML & $1,23 \pm 0,03$ & 24 & $2,59 \pm 0,01$ & 35 & $5,44 \pm 0,08$ & 35 & $1,53 \pm 0,07$ & 11 & $2,60 \pm 0,13$ & 15 & $5,40 \pm 0,15$ & 15 \\
\hline & AN & $1,21 \pm 0,03$ & 4 & $2,55 \pm 0,02$ & 5 & $5,62 \pm 0,11$ & 5 & $1,76 \pm 0,08$ & 4 & $2,55 \pm 0,03$ & 5 & $5,59 \pm 0,07$ & 5 \\
\hline & SCCB & $1,26 \pm 0,09$ & 3 & $2,58 \pm 0,03$ & 3 & $5,46 \pm 0,16$ & 3 & 1,33 & 1 & 2,55 & 1 & 5,60 & 1 \\
\hline & Total & $1,24 \pm 0,02$ & 49 & $2,57 \pm 0,02$ & 65 & $5,60 \pm 0,14$ & 65 & $1,54 \pm 0,03$ & 34 & $2,59 \pm 0,10$ & 41 & $5,45 \pm 0,07$ & 41 \\
\hline \multirow[t]{5}{*}{ Pabp } & VDRC & & 0 & 2,26 & 1 & 7,37 & 1 & - & 0 & & 0 & - & 0 \\
\hline & RSML & $1,09 \pm 0,02$ & 14 & $2,45 \pm 0,05$ & 16 & $6,32 \pm 0,34$ & 16 & $1,48 \pm 0,09$ & 4 & $2,34 \pm 0,10$ & 6 & $6,85 \pm 0,28$ & 6 \\
\hline & AN & $1,00 \pm 0,04$ & 13 & $2,43 \pm 0,03$ & 14 & $6,27 \pm 0,18$ & 14 & $1,59 \pm 0,10$ & 5 & $2,38 \pm 0,06$ & 6 & $6,55 \pm 0,06$ & 6 \\
\hline & SCCB & & 0 & - & 0 & - & 0 & - & 0 & & 0 & - & 0 \\
\hline & Total & $1,05 \pm 0,02$ & 27 & $2,43 \pm 0,03$ & 31 & $6,33 \pm 0,19$ & 31 & $1,54 \pm 0,07$ & 9 & $2,36 \pm 0,08$ & 12 & $6,70 \pm 0,16$ & 12 \\
\hline \multirow[t]{5}{*}{ Heas } & VDRC & $1,01 \pm 0,05$ & 4 & $2,81 \pm 0,06$ & 6 & $4,54 \pm 0,22$ & 6 & 1,35 & 1 & 2,88 & 1 & 4,25 & 1 \\
\hline & RSML & $0,96 \pm 0,02$ & 6 & $2,86 \pm 0,05$ & 8 & $4,35 \pm 0,16$ & 8 & - & 0 & 2,80 & 1 & 4,52 & 1 \\
\hline & AN & 0,98 & 1 & 3,07 & 1 & 3,67 & 1 & - & 0 & 2,97 & 1 & 3,95 & 1 \\
\hline & SCCB & 0,87 & 1 & 2,78 & 1 & 4,62 & 1 & $1,08 \pm 0,17$ & 2 & $2,84 \pm 0,02$ & 2 & $4,39 \pm 0,06$ & 2 \\
\hline & Total & $0,96 \pm 0,03$ & 12 & $2,84 \pm 0,03$ & 16 & $4,41 \pm 0,12$ & 16 & $1,17 \pm 0,13$ & 3 & $2,87 \pm 0,03$ & 5 & $4,30 \pm 0,10$ & 5 \\
\hline \multirow[t]{5}{*}{ Hehe } & VDRC & $0,73 \pm 0,06$ & 2 & $2,73 \pm 0,01$ & 2 & $4,80 \pm 0,03$ & 2 & & 0 & & 0 & - & 0 \\
\hline & RSML & $0,75 \pm 0,05$ & 5 & $2,85 \pm 0,04$ & 6 & $4,37 \pm 0,15$ & 6 & $1,22 \pm 0,06$ & 4 & $2,94 \pm 0,09$ & 5 & $4,12 \pm 0,29$ & 5 \\
\hline & AN & $0,71 \pm 0,06$ & 4 & $2,83 \pm 0,09$ & 4 & $4,46 \pm 0,32$ & 4 & - & 0 & & 0 & - & 0 \\
\hline & SCCB & & 0 & - & 0 & & 0 & 1,05 & 1 & 2,43 & 1 & 6,27 & 1 \\
\hline & Total & $0,73 \pm 0,03$ & 11 & $2,82 \pm 0,04$ & 12 & $4,47 \pm 0,13$ & 12 & $1,10 \pm 0,11$ & 5 & $2,84 \pm 0,11$ & 6 & $4,49 \pm 0,39$ & 6 \\
\hline \multirow[t]{5}{*}{ Hetb } & VDRC & 1,24 & 1 & 3,07 & 1 & 3,67 & 1 & - & 0 & - & 0 & - & 0 \\
\hline & RSML & $1,05 \pm 0,01$ & 2 & $3,13 \pm 0,01$ & 2 & $3,51 \pm 0,03$ & 2 & - & 0 & - & 0 & - & 0 \\
\hline & AN & 1,11 & 1 & 2,98 & 1 & 3,92 & 1 & - & 0 & - & 0 & - & 0 \\
\hline & SCCB & 0,88 & 1 & $3,13 \pm 0,06$ & 2 & $3,53 \pm 0,16$ & 2 & 1,45 & 1 & 3,17 & 1 & 3,41 & 1 \\
\hline & Total & $1,07 \pm 0,06$ & 5 & $3,09 \pm 0,03$ & 6 & $3,61 \pm 0,08$ & 6 & 1,45 & 1 & 3,17 & 1 & 3,41 & 1 \\
\hline
\end{tabular}

cima, enquanto os predadores aéreos voam rápido, mas no plano horizontal. Portanto, as borboletas quando realizam movimentos de manobrabilidade apresentam uma vantagem, pois permitem que elas escapem dos predadores (MARDEN \& C CHAI 1991), através de vôos rápidos e erráticos onde as borboletas devem ter velocidades al tas (HowLAND 1974).

A nálise de agrupamentos

Segundo Brower (1995) a atividade de locomoção nas borboletas não é um caráter que evoluiu devido à pressão de predadores, porque não é suficientemente similar entre os clados. Entretanto, as relações que são realizadas com os organismos e suas adaptações nem sempre são fruto de pressões sel etivas como normalmente são vistas. Muitas das características atuais existem por causa de pressões seletivas passadas. Podem existir outras razões para as variações observadas na natureza (WILLIAMS 1966).

$\mathrm{Na}$ figura 3 podem ser observados dois grandes grupos: um (A) formado pelas subespécies de Mimoides e $H$. hectorides, caracterizado por uma carga unitária média baixa (machos = $0,77 \mathrm{mg} / \mathrm{mm}^{2} \pm 0,03 \mathrm{mg} / \mathrm{mm}^{2}$; fêmeas $=1,04 \mathrm{mg} / \mathrm{mm}^{2} \pm 0,07$ 
$\mathrm{mg} / \mathrm{mm}^{2}$ ), índice de estreiteza médio intermediário (machos $=$ $2,70 \pm 0,08$; fêmeas $=2,65 \pm 0,12$ ) eárea al ar média igual mente baixa (machos $=1762 \mathrm{~mm}^{2} \pm 263 \mathrm{~mm}^{2}$; fêmeas $=1913 \mathrm{~mm}^{2} \pm$ $260 \mathrm{~mm}^{2}$ ) em relação as demais espécies estudadas; o outro (B) constituído por dois subgrupos. $O$ primeiro $\left(B_{1}\right)$ é formado somente por espécies de Troidini e o segundo $\left(B_{2}\right)$ por espécies de Papilionini. O primeiro subgrupo apresenta uma carga unitária média alta (machos $=1,16 \mathrm{mg} / \mathrm{mm}^{2} \pm 0,13 \mathrm{mg} / \mathrm{mm}^{2}$; fêmeas $\left.=1,55 \mathrm{mg} / \mathrm{mm}^{2} \pm 0,24 \mathrm{mg} / \mathrm{mm}^{2}\right)$, um índice de estreiteza médio baixo (machos =2,59 $\pm 0,19$; fêmeas $=2,59 \pm 0,22$ ) e uma área alar intermediária as demais borboletas estudadas (machos $2304 \mathrm{~mm}^{2} \pm 276 \mathrm{~mm}^{2}$; fêmeas $=2629 \mathrm{~mm}^{2} \pm 295 \mathrm{~mm}^{2}$ ). Um índice de estreiteza baixo determina uma asa com tendência a forma elíptica, capaz de sustentar um peso maior por unidade de área. Este tipo de asas pode decolar e acelerar o organismo mais rapidamente, proporcionando um vôo batido, com controle preciso e grande manobrabilidade (HILDEBRAND 1995, TeNnEKEs 1996), o que seria vantajoso em espaços reduzidos como florestas, por exemplo. Estas características podem ser atribuídas a Mimoides spp., $H$. hectorides e Troidini, que embora tenham o mesmo tipo de asa distinguem-se no tamanho por sustentarem um peso maior por unidade de área (carga unitária maior). O segundo subgrupo apresenta uma carga unitária média menor que o primeiro (machos $=1,02 \mathrm{mg} / \mathrm{mm}^{2}$ $\pm 0,08 \mathrm{mg} / \mathrm{mm}^{2}$; fêmeas $=1,29 \mathrm{mg} / \mathrm{mm}^{2} \pm 0,17 \mathrm{mg} / \mathrm{mm}^{2}$ ), um índice de estreiteza médio mais el evado que as demais espécies ( machos $=2,96 \pm 0,18$; fêmeas $=2,97 \pm 0,15$ ) e área das asas ampla (machos $=3814 \mathrm{~mm}^{2} \pm 727 \mathrm{~mm}^{2}$; fêmeas $=4438 \mathrm{~mm}^{2} \pm$ $391 \mathrm{~mm}^{2}$ ). Tais características determinam uma asa do tipo planadora (HILDEBRANd 1995, TenNekEs 1996), própria para deslocamentos longos, aos saltos, em locais abertos como os hábitats que estas borboletas ocupam, desenvolvendo velocidades médias mais baixas (machos $=4,98 \mathrm{~m} / \mathrm{s} \pm 0,65 \mathrm{~m} / \mathrm{s}$; fê meas $=6,06 \mathrm{~m} / \mathrm{s} \pm 1,29 \mathrm{~m} / \mathrm{s}$ ). Tais características são importantes se levarmos em conta os ambientes que estas borboletas podem ocupar, desde florestas até hábitats abertos.

Estes resultados mostram que Papilionini podem ter se diferenciado dos demais desenvolvendo asas com índice de estreiteza mais alto, produzindo asas mais longas e estreitas, que sustentam um peso menor por unidade de área, capazes de desenvolver velocidades elevadas, porém com batimentos lentos. Estas relações poderiam explicar também o vôo aos saltos e errático que al guns papilionídeos apresentam.

\section{CONCLUSÕES}

O tamanho corporal e os padrões aerodinâmicos fornecem informações importantes sobre a evolução dos papilionídeos. Os valores obtidos mostram quea massa torácica, o comprimento e a área das asas estão intimamente relacionados, e que os aumentos destes três parâmetros são diretamente proporcionais. Os resultados indicam também que as proporções se ajustam à massa final do corpo. Tanto a massa como as asas, contribuem para estabel ecer diferenças entre as espéciese os sexos. As espécies que apresentaram maior massa corporal e maior capacidade de vôo neste trabal ho são citadas na literatura como palatáveis. Em ambos os sexos a massa do tórax e o tamanho das asas são significativamente influenciados pelo hábitat. Conforme os hábitats se tornam mais antrópicos e com menor cobertura vegetal os valores médios se elevam.

A massa total, o comprimento e a largura são componentes importantes na determinação do tamanho e da forma das asas. Asas longas e estreitas estão associadas a índices de estreiteza mais el evados e asas curtas e largas a aumentos na massa total. As asas posteriores apresentaram maior variabilidade, cujas diferenças podem ser atribuídas ao papel que estas exercem na regulação da direção e manobrabilidade do vôo.

As espécies estudadas podem ser associadas a dois tipos básicos de asas: a) asas do tipo elíptica, capazes de desenvolver altas velocidades e b) asas do tipo planadora, mais longas e estreitas, próprias para deslocamentos longos.

\section{A GRA DECIMENTOS}

À CAPES pela bolsa PICD. Ao professor Gustavo Schwartz (Universidade da Campanha, São Gabriel, Rio Grande do Sul) pelas críticas e sugestões efetuadas e ao Departamento de Engenharia Rural da UFSM por ter cedido o programa SIDGER para efetuar as medidas nas asas.

\section{REFERÊNCIAS BIBLIOGRÁ FICAS}

BAKER, R.R. 1972. Territorial behavior of the nymphalid butterflies Aglais urticae and Inachis io. Journal of Animal Ecology, London, 41: 453-469.

BAKER, P.S.; M. GEWECKE, \& R.J. COOTER. 1981. The natural flight of the migratory locust, Locusta migratoria L. III. Wing-beat frequency, flight speed and altitude. Journal of Comparative Physiology, Heidelberg, 141: 233-237.

Balciunas, J. \& K. Knopf.1977. Orientation, flight speeds and tracks of three species of migrating butterflies. Florida Entomologist, Gainesville, 60: 37-39.

Betts, C.R. \& R.J. Wootton. 1988. Wing shape and flight behaviour in butterflies (Lepidoptera: Papilionoidea and Hesperioidea): a preliminary analysis. Journal of Experimental Biology, Cambridge, 138: 271-288.

BLAIR, R.B. \& A.E. LAUner. 1997. Butterfly diversity and human land use: species assemblages along an urban gradient. Biological Conservation, London, 80: 113-125.

BorGIA, G. 1981. Mate selection in the fly Scatophaga stercoraria: female choice in a male-controlled system. Animal Behavior, London, 29: 71-80.

BRodSKY, A.K. 1994. The evolution of insect flight. Oxford, Oxford University Press, XIV+229p.

BRower, A.V.Z. 1995. Locomotor mimicry in butterflies? A critical review of the evidence. Philosophical Transactions of The Royal Society of London Series B-Biological 
Sciences, London, 347: 413-425.

BROWER, L.P. \& J.V.Z. BRoWER. 1964. Birds, butterflies, and plant poisons: A study in ecological chemistry. Zoologica, NY, 49: 137-159.

BRown JR., K.S. 1992. Borboletas da Serra do Japi: diversidade, hábitats, recursos alimentares e variação temporal, p. 142186. In: L.P.C. M ORELLATO (Org.). Historia natural da Serra do Japi: ecologia e preservação de uma área florestal no sudoeste do Brasil. Campinas, Editora da UNICAMP/ FAPESP, 321p.

ChAl, P. \& R. DUdLEY. 1995. Limits to vertebrate locomotor energetics suggested by hummingbirds hovering in heliox. Nature, London, 377: 722-725.

CHAI, P. \& D. MILLARD. 1997. Flight and size constraints: hovering performance of large hummingbirds under conditions of maximal loading. Journal of Experimental Biology, Cambridge, 200: 2757-2763.

CHAI, P. \& R.B. SRYGLEY. 1990. Predation and the flight, morphology, and temperature of neotropical rain forest butterflies. American Naturalist, Chicago, 135: 748-765.

ChAl, P.; J.S.C. Chen \& R. DudLEY. 1997. Transient hovering performance of hummingbirds under conditions of maximal loading. Journal of Experimental Biology, Cambridge, 200: 921-929.

DAVIES, N.B. 1978. Territorial defense in the speckled wood butterfly (Pararge aegeria): the resident always wins. Animal Behavior, London, 26: 138-147.

DeVRIES, P.J. 1986. Hostplant records and natural history notes on Costa Rica butterflies (Papilionidae, Pieridae and Nymphalidae). Journal of Research on the Lepidoptera, Beverly Hills, 24: 290-333.

DeVRIES, P.J. \& R. Dudley. 1990. Morphometrics, airspeeds, thermoregulation and lipid reserves of migrating Urania fulgens (Uraniidae) moths in natural free flight. Physiological Zoology, Chicago, 63: 235-251.

Dudley, R. 1990. Biomechanics of flight in neotropical butterflies: morphometrics and kinematics. Journal of Experimental Biology, Cambridge, 150: 37-53.

. 2000. The biomechanics of insect flight. Princeton, Princeton University Press, $X I 1+476 p$.

Dudley, R. \& P.J. DeVries. 1990. Flight physiology of migrating Urania fulgens (Uraniidae) moths: kinematics and aerodynamics of natural freeflight. Journal of Comparative Physiology A, Heidelberg, 167: 145-154.

DudLey, R. \& C.P. ElLINGTON. 1990. Mechanics of forward flight in bumblebees. II. Quasi-steady lift and power requirements. Journal of Experimental Biology, Cambridge, 148: 53-88.

DudLEY, R. \& R.B. SRYGLeY. 1994. Flight physiology of Neotropical butterflies: allometry of airspeeds during natural free flight. Journal of Experimental Biology, Cambridge, 191: 125139.

Ehrlich, P.R.; D.E. Breedlove; P.F. Brussard \& M.A. Sharp. 1972. Weather and the regulation of subalpine populations.
Ecology, Washington, 53: 243-247.

ElLINGton, C.P. 1984. The aerodynamics of hovering insect flight. III. Kinematics. Philosophical Transactions of the Royal Society of London Series B, London, 305: 41-78.

Ellington, C.P.; K.E. Machin \& T.M. Casey. 1990. Oxygen consumption of bumblebees in forward flight. Nature, London, 347: 472-473.

EnNos, A.R. 1989. The kinematics and aerodynamics of the free flight of some Diptera. Journal of Experimental Biology, Cambridge, 142: 49-85.

FAIRBAIRN, D.J. \& D.A. RofF. 1990. Genetic correlation among traits determining migratory tendency in the sand cricket Gryllus firmus. Evolution, Philadel phia, 44: 1787-1795.

GILBERT, L.E. 1980. Food web organization and the conservation of neotropical diversity, p. 11-34. In: M.E. Soule \& B.A. WILCox (Eds). Conservation biology: an evolutionaryecological perspective, Sunderland, Sinauer Associates, $\mathrm{XV}+395 \mathrm{p}$.

1984. The biology of butterfly communities, p. 4154. In: R.I. VANE-W RIGHT \& P.R. ACKERY (Eds). The biology of butterflies. Princeton, Princeton University Press, XXIV+ $429 p$.

HaRRISON, R. 1980. Dispersal polymorphism in insects. Annual Review of Ecology and Systematics, Cambridge, 11: 95118.

HARTMAN, F.A. 1961. Locomotion mechanisms of birds. Smithsonian Miscellaneous Collections, Washington, 143: 191.

Hildebrand, M. 1995. Análise da estrutura dos vertebrados. São Paulo, Atheneu Editora, $X+700 p$.

HowLAND, H.C. 1974. Optimal strategies for predator avoidance: The relative importance of speed and maneuverability. Journal of Theoretical Biology, New York, 47: 333-350.

Johnson, C.G. 1969. Migration and Dispersal of Insects by Flight. London, Methuem, XXI+763p.

Kingsolver, J.G. 1985. Butterfly engineering. Scientific American, New York, 253: 106-113.

KLEIN, R.M. 1983. Aspectos fitofisionômicos da floresta estacional na fralda da Serra Geral (RS). In: Anais do Congresso Nacional de Botânica, Porto Alegre, 192p.

Kremen, C. 1992. Assessing the indicator properties of species assemblages for natural areas monitoring. Ecological applications, Washington, 2:203-217.

LEWIS, T. \& L.R. TAYLOR. 1967. Introduction to Experimental Ecology. London, Academic Press, 401p.

MARDEN, J.H. 1989. Effects of load-lifting constraints on the mating system of a dance fly. Ecology, Washington, 70: 496-502.

MARDEN, J.H. \& P. Chal 1991. Aerial predation and butterfly design: how palatability, mimicry, and the need for evasive flight constrain mass allocation. American Naturalist, Chicago, 137: 15-36.

MoRGAN, K.R.; T.E. SHELLYY \& L.S. KIMSEY. 1985. Body temperature 
regulation, energy metabolism and foraging in light-seeking and shade-seeking robber flies. Journal of Experimental Biology, Cambridge, 155: 561-570.

MurPhY, D.D.; K.E. FreAs \& S.B. Weiss. 1990. An environmentmetapopulation approach to population viability analysis for a threatened invertebrate. Conservation Biology, Boston, 4: 41-51.

Norberg, U.M. 1990. Vertebrate Flight: mechanics, physiology, morphology, ecology and evolution. Berlin, Springer-Verlang, 291p.

PALmer, J.O. \& H. Dingle. 1989. Responses of selection on flight behaviour in a migratory population of milkweed bug (Oncopeltus fasciatus). Evolution, Philadel phia, 43: 1805-1808.

Pinheiro, C.E.G. 1996. Palatability and escaping ability in Neotropical butterflies: tests with wild kingbirds (Tyrannus melancholicus, Tyrannidae). Biological Journal of the Linnean Society, London, 59: 351-365.

PYLE, R.M. 1980. Butterfly eco-geography and biological conservation in Washington. Atala, Ithaca, 8: 1-26.

RACheli, T. \& L. PARISET. 1992. II genere Battus - Tassonomia e Storia Naturale. Fragmenta Entomologica, Roma, 23 (Supl.): 1-150.

RoFf, D.A. 1986. The evolution of wing dimorphism in insects. Evolution, Philadelphia, 40: 1009-1021.

Schwartz, G. \&. R.A. Di Mare. 2001. Diversidade de quinze espécies de borboletas (Lepidoptera, Papilionidae) em sete comunidades de Santa Maria, RS. Ciência Rural, Santa Maria, 31 (1): 49-55.

SRYGLEY, R.B. \& P. ChAl. 1990. Flight morphology of Neotropical butterflies: palatability and distribution of mass to the thorax and abdomen. Oecologia, Berlin, 84: 491-499.

TenNekES, H. 1996. The Simple Science of Flight from Insects to Jumbo Jets. Cambridge, MT Press, $X+132 p$.

TYLeR, H.A.; K.S. BRown JR. \& K.H. WiLson. 1994. Swallowtail butterflies of the Americas: a study in biological dynamics, ecological diversity, biosystematics and conservation. Gainesville, Scientific Publishers, 376p.

VogeL, S. 1966. Flight in Drosophila. I. Flight performance of tethered flies. Journal of Experimental Biology, Cambridge, 44: 567-578.

WALOFF, Z. 1972. Observations on the airspeeds of freely flying locusts. Animal Behavior, London, 20: 367-372.

W ARD, J.H. 1963. Hierarchical grouping to optimize an objective function. Journal of the American Statististical Association, Alexandria US, 58: 236.

WatT, W.B.; F.S. CheW; L.R.G. Snyder; A.G. Watt \& D.E. RothschILD. 1968. Population structure of pierid butterflies, I. Numbers and movements of some montane Colias species. Oecologia, Berlin, 27: 1-22.

WeISS, S.B.; R.R. White; D.D. MURPhy \& P.R. EHRLICH. 1987. Growth and dispersal of larvae of the checkerspot butterfly Euphydryas editha. Oikos, Copenhagen, 50: 161-166.

Weiss, S.B.; D.D. Murphy \& R.R. White. 1988. Sun, slope and butterflies: topographic determinants of habitat quality for Euphydryas editha. Ecology, Washington, 69: 1486-1496.

WEIs-Fogh, T. 1956. Biology and physics of locust flight. II. Flight performance of the desert locust (Schistocerca gregaria). Philosophical Transactions of the Royal Society of London, Series B, London, 239: 459-510.

WILLIAMS, G.C. 1966. Adaptation and natural sel ection: A critique of some current evolutionary thought. New Jersey, Princeton University Press. Princeton, $X V+307 p$.

ZAR, J.H. 1999. Biostatistical Analysis. New Jersey, PrenticeHall, XII+663p.

Recebido em 02.III.2004; aceito em 04.XI.2004. 